\title{
ANÁLISE ESPACIAL DE ATRIBUTOS FÍSICOS E CARBONO ORGÂNICO EM ARGISSOLO VERMELHO-AMARELO CULTIVADO COM CANA-DE-AÇÚCAR ${ }^{1}$
}

\author{
Spatial analysis of physical attributes and organic carbon from yellow- \\ red alfissol with sugarcane crop \\ Joedna Silva $\mathrm{Cruz}^{2}$, Raimundo Nonato de Assis Júnior ${ }^{3}$, Sammy Sidney Rocha Matias ${ }^{4}$, \\ Jesús Hernan Camacho-Tamayo ${ }^{5}$, Rodrigo de Castro Tavares ${ }^{6}$
}

\begin{abstract}
RESUMO
A agricultura convencional utiliza o solo intensivamente, modificando os seus atributos. Neste estudo, objetivou-se avaliar a variabilidade espacial de alguns atributos físicos e carbono orgânico do solo em um Argissolo Vermelho-Amarelo cultivado com canade-açúcar, usando geoestatística. O trabalho foi realizado em Maracanaú - CE, em uma área de produção de cana-de-açúcar, manejado mediante preparo conservacionista sobre uma cobertura de palhada de cana-de-açúcar. As amostras de solo foram retiradas de uma profundidade de $0,00-0,20 \mathrm{~m}$, em uma malha, com intervalo regular de $10 \mathrm{~m}$, totalizando 100 pontos. Em cada amostra, foi analisado densidade de partículas, densidade do solo, carbono orgânico, porosidade total, macroposidade e microposidade. O coeficiente de variação indicou variabilidade baixa para densidade de partículas, densidade do solo e porosidade total e média para as variáveis macroporosidade, microporosidade e carbono orgânico. As variáveis analisadas mostraram dependência espacial, a qual foi observada nos mapas de krigagem. A distribuição de poros por tamanho e a porosidade total indicam condições físicas razoavelmente boas, embora com valores de densidade do solo ligeiramente acima do nível considerado adequado para a classe textural do solo.
\end{abstract}

Termos de indexação: Agricultura de precisão, geoestatística, densidade do solo, porosidade.

\begin{abstract}
Conventional farming uses the soil intensively, modifying its attributes. The goal of this study is to evaluate the spatial variability of some physical attributes and soil organic carbon in red-yellow Anfissol cultivated with sugar cane, using geostatistics. The work was carried out at Maracanaú - CE, in area with sugar cane production, managed by conservation tillage practices five years ago, which remained covered with sugarcane straw mulch. Soil samples were taken from 0.00 to $0.20 \mathrm{~m}$ de pth, in a mesh, with a regular interval of $10 \mathrm{~m}$, totaling 100 points. For each sample, soil particle density, bulk density, organic carbon, porosity, macro pores, and micro pores were analyzed. The coefficient of variation indicated low variability for soil particles density, bulk density, total and average porosity for the variables of macro- and microporosiy, and organic carbon. The variables analyzed showed spatial dependence, which was observed in kriging maps. The pores distribution by size and total porosity indicate adequate physical conditions, with bulk density values slightly above the level considered suitable for this textural class of soil.
\end{abstract}

Index terms: Precision agriculture, geostatistics, bulk density, porosity.

(Recebido em 30 de julho de 2008 e aprovado em 9 de setembro de 2008)

\section{INTRODUÇÃO}

A agricultura convencional considera as áreas de cultivo homogêneas, o que implica no emprego de práticas de manejo do solo uniformes, o que inclui a semeadura, as aplicações de fertilizantes e agrotóxicos, não levando em conta a variabilidade espacial do solo (McBratney \& Pringle, 1998). Essa variabilidade é gerada no tempo, pela intensidade dos processos formadores do solo (Burrough, 1993) e influenciada pela ação antrópica, particularmente pelo efeito das práticas agrícolas (Silveira et al., 2000). Conhecer a variabilidade do solo permite um melhor entendimento das relações entre seus atributos e os fatores ambientais (Goovaerts, 1998) e ajuda a determinar práticas de manejo específico para sua aplicação na produção de culturas (Plant, 2001). De fato, o reconhecimento da variabilidade tem conduzido a uma reavaliação do manejo convencional das culturas, e deve ser levada em consideração tanto em experimentos científicos, quanto em áreas destinadas à exploração comercial, para não incorrer em erros de planejamentos (Prevedello, 1987 e Silva et al.,2008). Entretanto, deve-se levar em conta que a

\footnotetext{
Parte da dissertação do primeiro autor apresentada ao Departamento de Ciências do Solo da Universidade Federal do Ceará. Projeto financiado pelo programa CAPES/PROCAD/ESALQ/UFC 0095/00-1.

${ }^{2}$ Universidade Federal do Ceará/UFC - Departamento de Fitotecnia - Fortaleza, CE

${ }^{3}$ Universidade Federal do Ceará/UFC - Departamento de Ciências do Solo - Fortaleza, CE

${ }^{4}$ Universidade Estadual Paulista Júlio de Mesquita Filho/FCAV/UNESP - Departamento de Solos e Adubos - Via de Acesso s/n - $14884-900$ - Jaboticabal, SP ymmsa2001@yahoo.com.br

${ }^{5}$ Universidade Nacional de Colômbia, Faculdade de Engenharia, Programa de Engenharia Agrícola, Sede Bogotá - Colômbia

UUniversidade Federal de Viçosa/UFV - Departamento de Solos - Viçosa,MG
} 
determinação da variabilidade requer uma amostragem precisa e confiável, para evitar erros graves de interpretação.

As propriedades físicas têm um papel importante na fertilidade e é um componente básico da qualidade do solo (Amézquita et al., 2004); sendo portanto, proposto o estudo desses atributos para definir zonas de manejo específico (Boruvka et al., 2002).

A densidade de partículas (Dp) e a densidade do solo (Ds) exercem papel preponderante na porosidade total e, por conseguinte, no movimento e armazenagem de água e ar no solo. A Dp depende da composição mineral e orgânica do solo, em que o manejo de culturas e, particularmente, o de materiais orgânicos pode conduzir a uma notória variabilidade espacial, até mesmo em uma área considerada homogênea quanto ao tipo de solo. A matéria orgânica, além de contribuir nos atributos químicos, na fertilidade e na biota do solo, tem um efeito positivo na conservação de atributos físicos. Segundo Cruz et al. (2003), a estabilidade da estrutura depende da matéria orgânica, verificando-se menor risco de degradação estrutural e de compactação em solos com maiores teores de carbono orgânico (CO), como acontece em áreas de plantio direto.

Em um estudo semelhante a este Oliveira et al. (2007), encontraram uma relação positiva da densidade do solo e macroposidade, tanto em superfície, quanto em subsuperfície, comprovando que quanto maior a densidade do solo menor a macroposidade.

Segundo Silva \& Kay (1997), a microporosidade do solo é fortemente influenciada pela textura, teor de carbono orgânico e pouco influenciada pelo aumento da densidade do solo, promovido pelo tráfego de máquinas e implementos. Em estudo comparativo das propriedades físicas de um Latossolo Vermelho distrófico, cultivado e sob mata nativa, Araujo et al. (2004), não verificaram diferenças significativas na microporosidade.

Considerando a importância da variabilidade dos solos, neste trabalho objetivou-se avaliar a variabilidade espacial das densidades do solo e de partículas, do carbono orgânico e da geometria porosa de um Argissolo Vermelho-Amarelo cultivado com cana-de-açúcar.

\section{MATERIAL E MÉTODOS}

O trabalho foi conduzido na Fazenda Jaçanaú, situada no município de Maracanaú - CE, cujas coordenadas pontuais são $03^{\circ} 49^{\prime} 88^{\prime}$ " de latitude sul e 38³8'19', de longitude oeste, com altitude média de $47 \mathrm{~m}$. O clima da região, segundo a classificação de Köppen, é do tipo AW', caracterizado por ser quente e úmido e com temperaturas superiores a $18^{\circ} \mathrm{C}$ no mês mais frio. Apresenta precipitação média de $1200 \mathrm{~mm}$, com chuvas concentradas no período de fevereiro a abril. O relevo é suave ondulado com declividade entre 2 e $5 \%$. O experimento localizou-se em uma área comercial de cana-de-açúcar, cultivada há mais de 20 anos, fertirrigada, com colheita manual e sem queima de palha durante os últimos cinco anos. O solo foi classificado como Argissolo Vermelho-Amarelo eutrófico, textura arenosa (EMBRAPA, 2006), com teores médios de $810 \mathrm{~g} \mathrm{~kg}^{-1}$ de areia, $100 \mathrm{~g} \mathrm{~kg}^{-1}$ de silte e $90 \mathrm{~g} \mathrm{~kg}^{-1}$ de argila.

Os solos foram amostrados em pontos distanciados de 10 m em uma área com dimensões de 100 x 100 m em duas direções perpendiculares entre si, totalizando 100 pontos. Em cada ponto, fez-se a coleta de amostras de solo com estrutura deformada e indeformada (com anel volumétrico de $67,49 \mathrm{~cm}^{3}$, com diâmetro de $0,047 \mathrm{~m} \mathrm{e} 0,03 \mathrm{~m}$ de altura), na camada de $0,00-0,20 \mathrm{~m}$.

As amostras coletadas foram encaminhadas para o Laboratório de Física do Solo da Universidade Federal do Ceará, para determinação da densidade de partículas e densidade do solo, segundo a metodologia descrita por Blake \& Hartge (1986a,b), e porosidade do solo (total, macro e microporosidade), de acordo com a metodologia empregada por Danielson \& Sutherland (1986), na qual as amostras foram saturadas durante $24 \mathrm{~h}$ e pesadas obtendose, assim, a massa do solo saturado. A microporosidade foi determinada em funil de Haines, submetendo-se as amostras saturadas à tensão de $6 \mathrm{kPa}$ para a drenagem da água; após o equilíbrio as amostras foram levadas à estufa, cuja temperatura foi mantida em $105^{\circ} \mathrm{C}$, até peso constante. A umidade do solo, base volume, no equilíbrio com a tensão aplicada corresponde ao volume de microporos. A macroporosidade foi determinada pela diferença entre a porosidade total e a microporosidade. No laboratório de química do Solo da Universidade Federal do Ceará, o Carbono Orgânico foi determinado seguindo a metodologia descrita pela EMBRAPA (1997).

Para a análise estatística, inicialmente realizou-se um estudo exploratório de dados, com o software Statistic (1999), calculando medidas de localização (média, mediana, mínimo e máximo), de variabilidade (coeficiente de variação$\mathrm{CV}$ ) e de tendência central (assimetria e curtose). Dessa maneira verificou-se a normalidade dos atributos avaliados, que segundo Diggle \& Ribeiro (2000), não é requisitos indispensável, sendo que aqueles atributos que se aproximam da distribuição normal proporcionam melhores predições, quando associada às técnicas de geoestatística Para a análise do CV, usou-se a classificação de Warrick \& Nielsen (1980), com variabilidade baixa para valores menores de $12 \%$, média para valores entre 12 e $60 \%$, e alta para valores maiores de $60 \%$. 
Para determinar a variabilidade espacial, considerouse a teoria das variáveis regionalizadas, a qual dispõe de diferentes métodos de análise de variação espacial, sendo uma delas o semivariograma (Vieira, 2000). Os semivariogramas de cada atributo foram obtidos mediante $o$ programa GS ${ }^{+}$(Robertson, 1998). Por meio destes modelos, foi feita a predição de cada atributo em zonas não amostradas mediante krigagem, representados em mapas de contorno, utilizando o programa Surfer.(2000). Conjuntamente com os mapas e a análise de correlação linear de Pearson, verificouse a correlação entre os atributos do solo. A classificação do grau da dependência espacial (GDE) foi feita com base na razão entre o efeito pepita e o patamar $\left(\mathrm{C} / \mathrm{C}_{\mathrm{o}}+\mathrm{C}\right)$, sendo considerada forte para GDE superior de $75 \%$, moderada para GDE entre $25 \%$ e $75 \%$, e fraca para GDE inferior de $25 \%$ (Cambardella et al., 1994).

\section{RESULTADOS E DISCUSSÃO}

Os valores de média e mediana foram semelhantes, e a assimetria e a curtose valores próximos de zero, indicando uma aproximação da distribuição normal (Tabela 1), como verificado pelo teste de Kolmogorov-Smirnov (K-S), o qual foi significativo para todos os atributos analisados. Resultados semelhantes foram obtidos para atributos físicos do solo por Souza et al. (2004) e Ramírez-López et al. (2008) e para CO por Bocchi et al. (2000).

Os valores médios obtidos de Ds, Dp e PT correspondem aos encontrados em estudos realizados por Ribeiro, et al. (2007a), para solos com altos teores de areia e, segundo Kiehl (1979), a PT está no intervalo característico dos solos arenosos e promovendo uma aeração ótima, com uma macroporosidade entre 20 e $30 \%$. A colheita manual pode ter favorecido a preservação da PT, em razão de uma menor frequência de tráfego de maquinaria agrícola na área de estudo.

A Dp apresentou valores levemente inferiores aos comumente obtidos para argissolos (Ribeiro et al., 2007b), possivelmente decorrente do acúmulo de resíduos vegetais na superfície do solo. Paiva et al. (2000) e Mota (2004), observaram valores semelhantes de Dp em outros Argissolos e Colet et al. (2009) em Latossolo.

Pelos valores de coeficiente de variação, verificam-se as variáveis avaliadas apresentaram de baixo a médio $\mathrm{CV}(<$ $12 \%$ e entre 12 e 60\%), concordando com Amaro Filho et al. (2007) e Ramírez-López et al. (2008). A macroporosidade e a microporosidade apresentaram variabilidade média, notandose que, nesse tipo de solo predominam os macroporos, situação típica em solos arenosos. $\mathrm{O}$ carbono orgânico apresentou o maior valor de $\mathrm{CV}$, com uma variabilidade média, concordando com Bocchi et al. (2000) e Muñoz et al. (2006).

Os ajustes dos modelos de semivariogramas mostraram que o único atributo que apresentou efeito pepita puro foi a microporosidade (Tabela 2), ou seja, uma distribuição aleatória na zona de estudo e independência espacial. Os outros parâmetros ajustaram-se aos modelos exponencial e gaussiano, concordando com Souza et al. (2004), Amaro Filho et al. (2007) e Ramírez-López et al. (2008). $\mathrm{O}$ grau de dependência espacial (GDE) foi moderado para todos os atributos que apresentaram ajuste a modelos de semivariogramas. $\mathrm{O}$ carbono orgânico foi o atributo que apresentou melhor ajuste $\left(R^{2}=0,97\right)$, seguido da Ds $\left(R^{2}=0,86\right)$ (Figura 1), os maiores valores de GDE, obtiveram os melhores ajustes na validação cruzada, com coeficientes de regressão (CRVC) mais próximos de um (Tabela 2). A Dp do solo foi o atributo que apresentou os menores valores de GDE e CRVC.

Tabela 1 - Medidas descritivas dos dados de densidade do solo (Ds), densidade de partículas (Dp), carbono orgânico (CO), porosidade total (PT), macroporosidade (MACRO) e microporsidade (MICRO).

\begin{tabular}{|c|c|c|c|c|c|c|}
\hline \multirow{2}{*}{ Atributo } & Ds & Dp & $\mathrm{CO}$ & PT & MACRO & MICRO \\
\hline & \multicolumn{2}{|c|}{--------g cm ${ }^{-3}$--------- } & $\mathrm{g} \mathrm{kg}^{-1}$ & \multicolumn{3}{|c|}{--------------------- \% ------------------------- } \\
\hline Média & 1,53 & 2,49 & 5,37 & 39,39 & 25,30 & 13,93 \\
\hline Desvio padrão & 0,07 & 0,07 & 1,02 & 3,00 & 3,13 & 2,45 \\
\hline Mediana & 1,53 & 2,49 & 5,42 & 39,61 & 25,92 & 13,54 \\
\hline $\mathrm{CV}(\%)$ & 4,58 & 2,81 & 18,95 & 7,61 & 12,35 & 17,59 \\
\hline Mínimo & 1,38 & 2,34 & 3,59 & 33,33 & 18,67 & 7,59 \\
\hline Máximo & 1,66 & 2,65 & 7,98 & 45,88 & 30,77 & 19,25 \\
\hline Assimetria & $-0,18$ & 0,26 & 0,12 & $-0,10$ & $-0,37$ & 0,18 \\
\hline Curtose & $-0,88$ & $-0,30$ & $-0,22$ & $-0,87$ & $-0,98$ & 0,27 \\
\hline $\mathrm{K}-\mathrm{S}$ & $0,85^{*}$ & $1,26^{*}$ & $0,81 *$ & $0,69 *$ & $1,14^{*}$ & $0,69 *$ \\
\hline
\end{tabular}

\footnotetext{
${ }^{(*)}$ significativo a $5 \%$ pelo teste de Kolmogorov-Smirnov (K-S).
} 
Tabela 2 - Parâmetros dos modelos de semivariogramas ajustados de densidade do solo (Ds), densidade de partículas (Dp), carbono orgânico (CO), porosidade total (PT), macroporosidade (MACRO) e microporosidade (MICRO).

\begin{tabular}{cccccccc}
\hline Atributo & Modelo & $\mathrm{C}_{0}$ & $\mathrm{C}_{0}+\mathrm{C}$ & $\mathrm{GDE}$ & Alcance (a) (m) & $\mathrm{R}^{2}$ & CRVC \\
\hline $\mathrm{Ds}$ & Exponencial & 0,00 & 0,01 & 0,58 & 12,58 & 0,86 & 0,90 \\
$\mathrm{Dp}$ & Gaussiano & 0,00 & 0,01 & 0,51 & 98,80 & 0,78 & 0,57 \\
$\mathrm{CO}$ & Gaussiano & 0,40 & 1,25 & 0,68 & 55,90 & 0,97 & 1,05 \\
PT & Exponencial & 5,95 & 12,14 & 0,51 & 97,90 & 0,72 & 0,85 \\
MACRO & Exponencial & 5,17 & 11,07 & 0,53 & 41,53 & 0,76 & 0,80 \\
MICRO & EPP & 5,46 & & & & &
\end{tabular}

$\mathrm{C}_{0}=$ efeito pepita; $\mathrm{C}_{0}+\mathrm{C}=$ patamar; $\mathrm{R}^{2}=$ coeficiente de determinação do modelo; $\mathrm{CRVC}=$ coeficiente de regressão da validação cruzada; $\mathrm{EPP}=$ efeito pepita puro, $\mathrm{GDE}\left(\mathrm{C}_{0} / \mathrm{C}_{0}+\mathrm{C} * 100\right)=$ grau de dependência espacial.
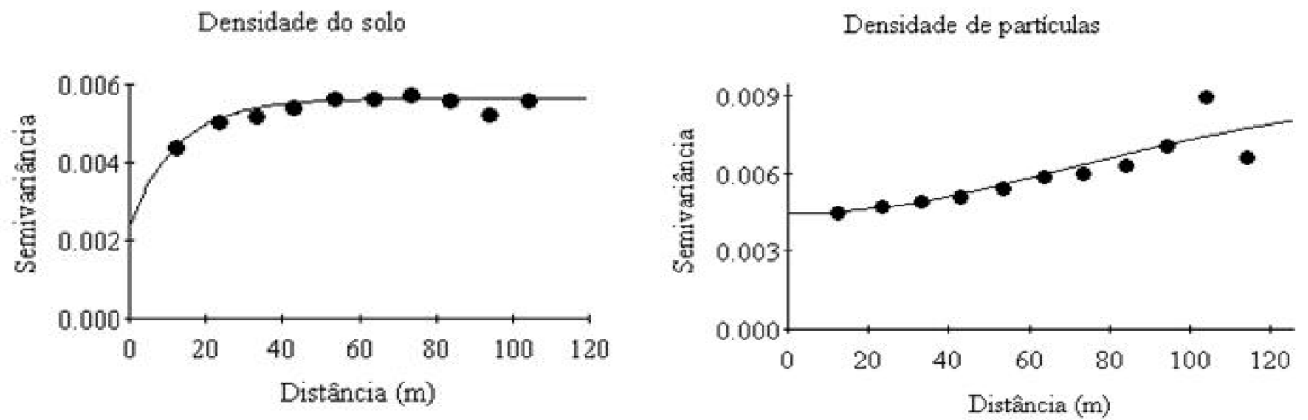

Carbono orgầnico

Porosidade total
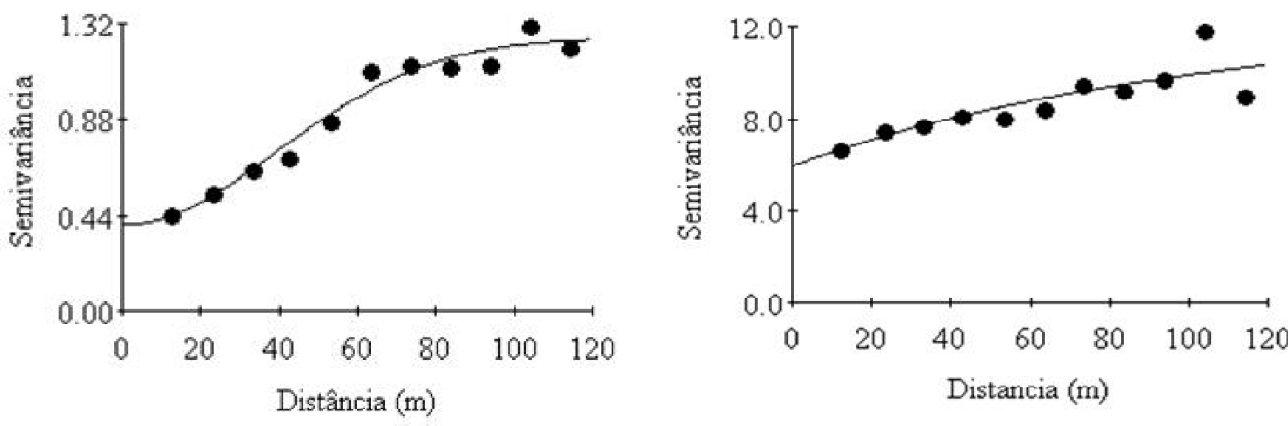

Macroporosidade
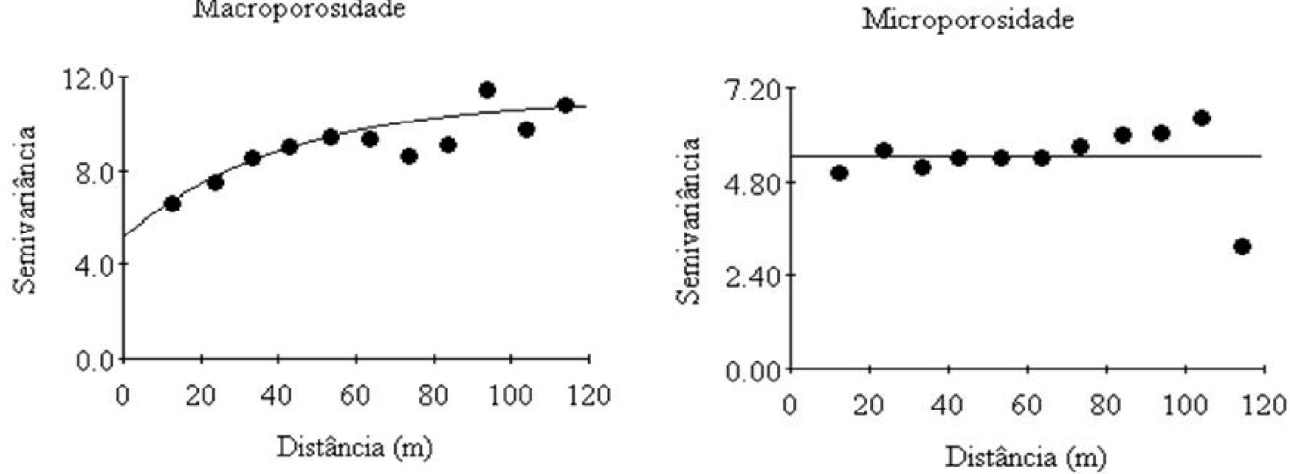

Figura 1 - Semivariogramas dos atributos estudados do solo: densidade do solo, densidade de partículas, carbono orgânico, porosidade total, macroporosidade e microporsidade. 
Em termos gerais, o comportamento do GDE dos atributos pode ser decorrente da intervenção antrópica, pela inserção da cultura da cana-de-açúcar. Segundo Cambardella et al. (1994), uma dependência espacial forte dos atributos do solo é atribuída a fatores intrínsecos (material de origem, relevo, clima, organismos e tempo), e uma dependência espacial fraca pode ser atribuída a fatores extrínsecos (adubação, calagem, preparo do solo, tráfego de maquinaria agrícola, dentre outros).

Todos os valores de alcance dos atributos dos solos tiveram um comportamento dentro da teoria das variáveis regionalizadas, sendo estes valores (Tabela 2) inferiores à máxima distância de amostragem (141,42 m) e o máximo Lag $(127,28 \mathrm{~m})$, o qual corresponde a $90 \%$ da distância máxima de amostragem (Robertson, 1998). O alcance fornece informações importantes para planejamento e avaliação experimental, além de subsidiar informações sobre a gênese e evolução dos solos na paisagem e, limites entre classes taxonômicas de solos (Souza et al., 2007)"

O CO apresentou correlação negativa com a Dp (Tabela 3), o qual pode ser verificado na Figura 2, onde áreas com maior teor de $\mathrm{CO}$ correspondem com as áreas de menor Dp. Segundo Kiehl (1979), a presença de matéria orgânica altera consideravelmente a Dp e a Ds.
As Ds e Dp não apresentaram correlações significativas entre si, como também é reportado por outros autores (Ramírez-López et al., 2008), mas estas se correlacionaram significativamente com porosidade total, macro e microposidade. Observa-se que áreas com maior Ds, correspondem a áreas de menor PT e menor macroporosidade (Figura 2), como também foi encontrado por Ramírez-López et al. (2008). Assim como, áreas de maior Dp correspondem a áreas de maior PT e maior macroposidade. Kulli et al. (2003) estabeleceram que solos submetidos a maior compactação pelo tráfego de maquinaria, reduz a macro e a PT e ainda, a aumenta Ds significativamente.

A PT apresentou correlação positiva com a macroporosidade, o qual é verificado nos mapas destes atributos (Figura 2). Esse fato deve-se à predominância dos macroporos sobre os microporos, como observado na Tabela 1. A microporosidade apresentou correlações lineares significativas com todos os atributos do solo estudados, com exceção do CO. Verifica-se também alta variabilidade espacial da microporosidade que não permitiu verificar uma correlação espacial com os outros atributos, decorrente do fato de apresentar independência espacial.

Tabela 3 - Correlação linear dos atributos densidade do solo (Ds), densidade de partículas (Dp), carbono orgânico (CO), porosidade total (PT), macroporosidade (MACRO) e microporosidade (MICRO).

\begin{tabular}{lcrrrrr}
\hline \multicolumn{1}{c}{ Atributo } & CO & Ds & Dp & PT & MACRO & MICRO \\
\hline CO & 1 & 0,09 & $-0,19$ & $-0,19$ & $-0,17$ & $-0,03$ \\
DS & & 1 & 0,15 & $-0,79^{* *}$ & $-0,42^{* *}$ & $-0,45^{* *}$ \\
DP & & & 1 & $0,48^{* *}$ & $0,50^{* *}$ & $-0,04^{* *}$ \\
PT & & & & $0,69^{* *}$ & $0,38^{* *}$ \\
MACRO & & & & 1 & $-0,41^{* *}$ \\
MICRO & & & & & 1 \\
\hline
\end{tabular}

**A correlação é significativa ao nível 0,01 de probabilidade. 

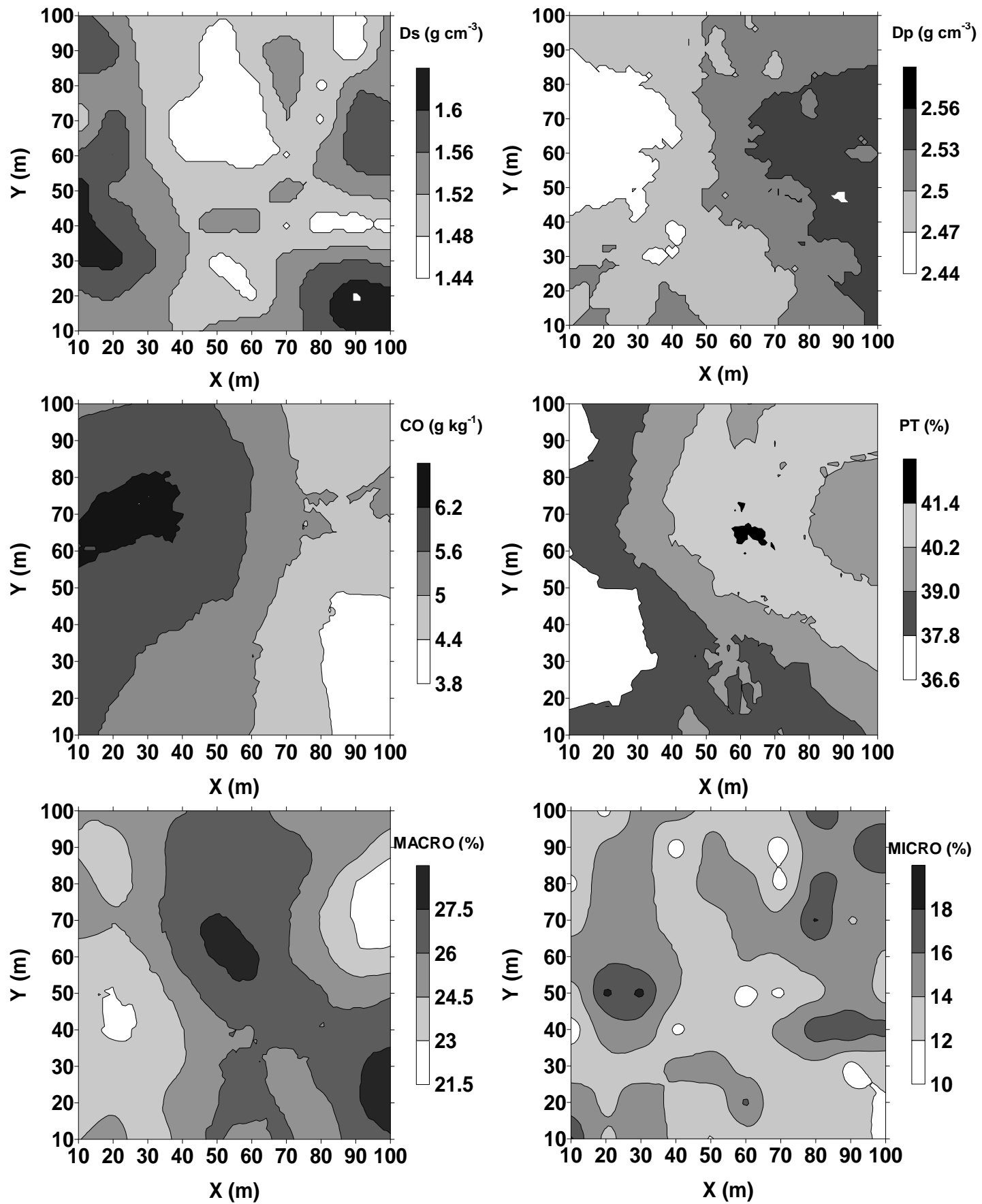

Figura 2 - Distribuição espacial das variáveis estudadas: densidade de partículas, densidade do solo, carbono orgânico, porosidade total, macroporosidade e microporosidade.

\section{CONCLUSÕES}

As variáveis analisadas indicaram estrutura de dependência espacial, o que permitiu o seu mapeamento, utilizando-se técnicas de geoestatística.
O uso da geoestatística e construção de mapas, a partir da krigagem, permitem identificar zonas de manejo, sendo, portanto, ferramentas adequadas para a tomada de decisões. 


\section{REFERÊNCIAS BIBLIOGRÁFICAS}

AMARO FILHO, J.; NEGREIROS, R.F.D.; ASSIS JÚNIOR, R.N.; MOTA, J.C.A. Amostragem e variabilidade espacial de atributos físicos de um Latossolo vermelho em Mossoró, RN. Revista Brasileira de Ciência do Solo, Viçosa, v.31, n.3, p.415-422, 2007.

AMÉZQUITA, E.; THOMAS, R.J.; RAO, I.M.; MOLINA, D.L.; HOYOS, P. Use of deep-rooted tropical pastures to build-up an arable layer through improved soil properties of an Oxisol in the Eastern Plains (Llanos Orientales) of Colombia. Agriculture, Ecosystems and Environment, Amsterdam, v.103, n.2, p.269-277, 2004.

ARAUJO, M.A.; TORMENA, C.A.; SILVA, A.P. Propriedades físicas de um latossolo vermelho distrófico cultivado e sob mata nativa. Revista Brasileira de Ciência do Solo, Viçosa, v.28, n.2, p.337-345, 2004.

BLAKE, G.R.; HARTGE, K.H. Bulk density. In: KLUTE, A. (Ed.). Methods of soil analysis. 2.ed. Madison: American Society of Agronomy, 1986a. part 1, p.363-375.

BLAKE, G.R.; HARTGE, K.H. Particle density. In: KLUTE, A. (Ed.). Methods of soil analysis. 2.ed. Madison: American Society of Agronomy, 1986b. part 1, p.377-382.

BOCCHI, S.; CASTRIGNANO, A.; FORNARO, F.; MAGGIORE, T. Application of factorial krigging for mapping soil variation at field scale. European Journal of Agronomy, Amsterdam, v.13, n.4, p.295-308, 2000.

BORÙVKA, L.; DONÁTOVÁ, H.; N-MEÈEK, K. Spatial distribution and correlation of soil properties in a field: a case study. Rostlinná Výroba, Prague, v.48, n.10, p.425432, 2002.

BURROUGH, P.A. Soil variability: a late 20th century view. Soils and Fertilizers, New York, v.56, n.5, p.529-562, 1993.

CAMBARDELLA, C.A.; MOORMAN, T.B.; NOVAK, J.M.; PARKIN, T.B.; KARLEN, D.L.; TURCO, R.F.; KONOPKA, A.E. Field-scale variability of soil properties in Central Iowa Soils. Soil Science Society American Journal, Madison, v.58, n.5, p.1501-1511, 1994.

COLET, M. J.; SVERZUT, C. B.; NETO, P. H. W.; SOUZA, Z. M. de. Alteração em atributos físicos de um solo sob pastagem após escarificação. Ciência e Agrotecnologia, Lavras, v. 33, n. 2, p. 361 - 368, mar./abr.; 2009.
CRUZ, A.C.R.; PAULETTO, E.A.; FLORES, C.A.; SILVA, J.B. Atributos físicos e carbono orgânico de um Argissolo Vermelho sob sistemas de manejo. Revista Brasileira de Ciência do Solo, Viçosa, v.27, n.6, p.11051112, 2003.

DANIELSON, R.E.; SUTHERLAND, P.L. Porosity. In: KLUTE, A. (Ed.). Methods of soil analysis. 2.ed.

Madison: American Society of Agronomy, Soil Science Society of America, 1986. part 1, p.443-461.

DIGGLE, P.J.; RIBEIRO, J.R. Model based geostatistics. São Paulo: Associação Brasileira de Estatística, 2000. $129 \mathrm{p}$.

\section{EMPRESA BRASILEIRA DE PESQUISA}

AGROPECUÁRIA. Manual de métodos de análises do solo. 2.ed. Rio de Janeiro: Ministério da Agricultura e do Abastecimento, 1997. 212p.

\section{EMPRESA BRASILEIRA DE PESQUISA}

AGROPECUÁRIA. Serviço de Produção de Informação. Sistema brasileiro de classificação de solo. Brasília, 2006. 412p.

GOOVAERTS, P. Geostatistical tools for characterizing the spatial variability of microbiological and physicochemical soil properties. Biology and Fertility of Soils, Berlin, v.27, n.4, p.315-334, 1998.

KIEHL, E.J. Manual da edafologia: relações solo-planta. São Paulo: Agronômica Ceres, 1979. 264p.

KULLI, B.; GYSI, M.; FLUHLER, H. Visualizing soil compaction based on flow pattern analysis. Soil and Tillage Research, Amsterdam, v.70, n.1, p.29-40, 2003.

McBRATNEY, A.B.; PRINGLE, M.J. Spatial variability in soil-implications for precision agriculture. In: STAFFORD, J.V. (Ed.). Precision agriculture: spatial variability in soil and crop. Warwick: BIOS, 1998. v.1, p.3-31

MOTA, J.C.A. Características físicas, químicas e minerais como suporte para o manejo dos principais solos explorados com a cultura do melão na chapada do Apodi - RN. 2004. 96f. Dissertação (Mestrado em Solos e Nutrição de Plantas)-Universidade Federal do Ceará, Fortaleza, 2004. 
MUÑOZ, J.D.; MARTÍNEZ, L.J.; GIRALDO, R. Varialilidad espacial de propiedades edáficas y su relación com el rendimiento em um cultivo de papa (Solanum tuberosum L.). Agronomia Colombiana, Bogotá, v.24, n.2, p.355-366, 2006.

OLIVEIRA, GC.; SEVERIANO, E.C.; MELLO, C.R. Dinâmica da resistência à penetração de um Latossolo Vermelho da microregião de Goiânia, GO. Revista Brasileira de Engenharia Agrícola Ambiental, Campina Grande, v.11, n.3, p.265-270, 2007.

PAIVA, A.Q.; SOUZA, L.S.; RIBEIRO, A.C.; COSTA, L.M. Propriedades físico-hídricas de solos de uma topossequiência de tabuleiro do Estado da Bahia. Pesquisa Agropecuária Brasilera, Brasilia, v.35, n.11, p.2295-2302, 2000.

PLANT, R.E. Site-specific management: the application of information technology to crop production.

Computers and Electronics in Agriculture, Amsterdam, v.30, n.1/3, p.9-29, 2001.

PREVEDELLO, B.M.S. Variabilidade especial de parâmetros de solo e planta. 1987. 166f. Tese (Doutorado em Solos e Nutrição de Plantas)-Escola Superior de Agricultura “Luís de Queiroz”, Piracicaba, 1987.

RAMIREZ-LÓPEZ, L.; REINA-SÁNCHEZ , A.; CAMACHO-TAMAYO, J.H. Variabilidad espacial de atributos físicos de un typic haplustox de los llanos orientales de Colômbia. Engenharia Agrícola, Jaboticabal, v.28, n.1, p.55-63, 2008.

RIBEIRO, K.A.; OLIVEIRA, T.S.; MENDONÇA E. de S.; XAVIER, F.A.S.; MAIA, S.M.F.; SOUSA, H.H.F.

Qualidade do solo na cultura do cajueiro anão precoce cultivado sob diferentes sistemas de manejo. Revista Brasileira de Ciência do Solo, Viçosa, v.31, n.2, abr. 2007b.

RIBEIRO, K.D.; MENEZES, S.M.; MESQUITA, M.G.B.F.; SAMPAIO, F.M.T. Propriedades físicas do solo, influenciadas pela distribuição de poros, de seis classes de solos da região de lavras - MG. Ciência e Agrotecnologia, Lavras, v.31, n. 4, p.1167-1175, 2007 a.

ROBERTSON, G.P. GS+ geostatistics for the environmental sciences: GS+ user's guide. Plainwell: Gamma, 1998. 152p.
SILVA, A.P.; KAY, B.D. Estimating the least limiting water range of soils from properties and management. Soil Science Society of America Journal, Madison, v.61, n.3, p.877-883, 1997.

SILVA, F. M. da; SOUZA, Z. M. de; FIGUEREDO, C. A. P. de; VIERA, L. H. de S.; OLIVEIRA E. de. Variabilidade espacial de atributos químicos e produtividade da cultura do café em duas safras agrícolas. Ciência e Agrotecnologia, Lavras, v. 32, n. 1, p.231-241, jan./fev., 2008.

SILVEIRA, P.M.; ZIMMERMAN, F.J.P.; SILVA, S.C. da; CUNHA, A.A. Amostragem e variabilidade espacial de características químicas de um latossolo submetido a diferentes sistemas de preparo. Pesquisa Agropecuária Brasileira, Brasilia, v.35, n.10, p.20572064, 2000.

SOUZA, Z. M. de; BARBIERI, D. M.; JÚNIOR, J. M.; PEREIRAG. T.; CAMPOS, M. C. C. Influência da variabilidade espacial de atributos químicos de um latossolo na aplicação de insumos para cultura de canade-açúcar. Ciência e Agrotecnologia, Lavras, v. 31, n. 2, p. 371-377, mar./abr., 2007.

SOUZA, Z.M.; MARQUES JÚNIOR, J.; PEREIRA, G.T.; BENTO, M.J.C. Variabilidade espacial de atributos físicos de um Latossolo Vermelho sob cultivo de canade-açúcar. Revista Brasileira de Engenharia Agrícola e Ambiental, Campina Grande, v.8, n.1, p.51-58, 2004.

STATISTIC. Basic statistics and tables. 1999. Disponível em: 〈www.statsoft.com〉. Acesso em: 10 mar. 2007.

SURFER FOR WINDOWS. Contouring and 3D surface mapping for scientist's engineers: user's guide. Realese 8.0. New York: Golden Software, 2000. Software.

VIEIRA, S.R. Geoestatística em estudos de variabilidade espacial do solo. In: NOVAIS, P.F.; ALVAREZ, V.H.; SCHAEFER, C.E.G.R. (Eds.). Tópicos em ciência do solo. Viçosa, MG: Sociedade Brasileira de Ciência do Solo, 2000. v.1, p.1-54.

WARRICK, A.W.; NIELSEN, D.R. Spatial variability of soil physical PToperties in the field. In: HILLEL, D. (Ed.). Applications of soil physics. New York: Academic. 1980. p.319-344. 